\title{
Oil-water separation efficiency and fluid mechanics of a hydrocyclone Xiangzhong Fan $^{1, a}$ \\ ${ }^{1}$ School of Petroleum Engineering, China University of Petroleum, Beijing 102249, China; afanxiangzhong_2016@163.com
}

Keywords: Computational fluid dynamics; oil-water separation; fluid mechanics; hydrocyclone;

\begin{abstract}
Hydrocyclone separation technology as a kind of effective oil water separation technology, has been applied in the oil industry. The paper proposes numerical simulation of computational fluid dynamics which combine Reynolds stress model and Euler multi-phase model together, and discusses the operating conditions and material properties making influence on the efficiency of oil-water separation of a hydrocyclone by Fluent6.3. The simulation shows that the hydrocyclone with diameter $50 \mathrm{~mm}$, two inlets, and a single cone angle $5.5^{\circ}$, has an optimum distribution ratio $10 \%$, a best concentration of oil droplets $0.5 \%$. Under the above conditions, the hydrocyclone could remove more than $80 \%$ per $15^{\mu \mathrm{m}}$ oil droplets, and the separation of oil droplets cut size is $9.2^{\mu \mathrm{m}}$ for $50 \%$ separation efficiency. Through simulated prediction of separation efficiency corresponding to the measured values, the error is obtained less than $15 \%$.
\end{abstract}

\section{Introduction}

Hydrocyclone separation technology, as a kind of effective oil water separation technology, has been applied in the oil industry due to the compact equipment without adding chemical agent[1-2]. Although the structure of Hydrocyclone separator is simple, many parameters variable affect the separation efficiency, such as import flow velocity, split ratio, oil droplet size, oil droplet concentration and so on. So many people do the experiments, in order to optimize parameters of the cyclone separator structure and operating conditions[3-5]. Because of many relevant variable parameters, there are many problems, such as workloads of experimental research, high cost and uncertainty on industrial amplification[6].

With the rapid development of computer technology, computational fluid dynamics method has become an important means for hydrocyclone due to the high speed and low cost[7-10]. Research of hydrocyclone separation mainly concentrated on solid-liquid aspect. The literature[11] proposed hydrocyclone with different diameter of spigots corresponding to the curve of separation gravel. The literature[12] simulated separation performance for $50 \mathrm{~mm}$ hydrocyclones and $76 \mathrm{~mm}$ hydrocyclones for ash by computational fluid dynamics. The literature[13] utilized computational fluid dynamics to optimize the structure of the hydrocyclones. As for oil-water separation, The literature[2] simulated dehydrate efficiency of different diameter of oil. But there are little research on operating conditions and material properties for dehydrate efficiency by computational fluid dynamics.

A full glass cyclone separator was established to make oil-water separation. Dynamic particle analysis instrument is used to analysis the flow field inside cyclone and droplet distribution[14]. In this paper, operating conditions including inlet velocity and separation ratio and material properties including droplet size and concentration of oil droplets have influence on dehydrate efficiency. Statistical method was used to study the relationship between the oil-water separation efficiency and operating parameters or physical properties of the hydrocyclone, which provides the basis for promoting the application of cyclone in oil-water separation. 


\section{Model Establishment}

\section{A. Parameters of Hydrocyclone}

The oil-water cyclone separator is a single cone with double inlet cyclone. The parameters of the cyclone separator are as Table.1.

Table 1 Parameters of the cyclone separator

\begin{tabular}{cc}
\hline Parameters & number \\
\hline Cyclone diameter & $50 \mathrm{~mm}$ \\
Cylindrical length & $50 \mathrm{~mm}$ \\
Inlet diameter & $13 \mathrm{~mm}$ \\
Underflow pipe diameter & $19 \mathrm{~mm}$ \\
Underflow pipe length & $900 \mathrm{~mm}$ \\
Overflow diameter & $8 \mathrm{~mm}$ \\
Taper angle & $5.5^{\circ}$ \\
Taper length & $322 \mathrm{~mm}$ \\
\hline
\end{tabular}

\section{B. Model Arithmetic}

Use commercial Fluent 6.3 software to simulate. Adopt continuity equation and Navier-Stokes equation, and import Reynolds Stress Model to get a solution. Because oil-water separation refer to two-phase fluid, so this paper utilizes Mixed Euler Equation[15], which assumes that all of the phase with the same stress field. As for any phase, we solve the momentum equation and continuity equation.

Operating parameters and material properties as two variable parameters in operation process. Operating parameters are inlet flow rate and split ratio. Among them, inlet flow rate correspond to fluid entering speed $4.18 \sim 20.93 \mathrm{~m} / \mathrm{s}$, where split ratio refer to the ratio of excessive traffic and inlet flow rate. Material parameters is the ratio of oil droplet size and concentration of oil droplets. During the process of simulation, when a parameter change, other parameters stay constant. Specific parameters are as follow Table.2.

Table 2.Parameters of the cyclone separator

\begin{tabular}{ccccc}
\hline & \multicolumn{2}{c}{ Operating Parameter } & \multicolumn{2}{c}{ Material Parameters } \\
\cline { 2 - 5 } Value & Inlet Flow Rate & Split Ratio & Oil Droplet Size & $\begin{array}{c}\text { Concentration of Oil } \\
\text { Droplets }\end{array}$ \\
\cline { 2 - 5 } & $2000 \sim 10000 \mathrm{~L} / \mathrm{h}$ & $2 \% \sim 20 \%$ & $5 \sim 30 \mathrm{um}$ & $0.05 \% \sim 5 \%$ \\
\hline
\end{tabular}

Use pressure-based solver and separation method to simulate and calculate. The grid is unstructured grids. Due to the complexity of flow field within the cyclone, it is difficult to ensure flow direction and the grid to be consistent. In order to solve this problem, the discrete method of the simulation control equation use second-order upwind scheme. Entrance is for speed, export is for pressure, and wall is for non-slip boundary. while the density of water phase is $1000 \mathrm{~kg} / \mathrm{m} 3$, the viscosity is $0.001003 \mathrm{~kg} / \mathrm{ms}$, the density of oil phase is $860 \mathrm{~kg} / \mathrm{m} 3$, and the viscosity is $0.0033 \mathrm{~kg} / \mathrm{ms}$.

\section{Results and Discussion}

\section{A. The effect of split ratio on separation efficiency}

Figure 1 simulate the effect of split ratio on separation efficiency and inlet pressure with $23 \mu \mathrm{m}$ oil droplets size under different inlet flow rate and different oil concentration. When inlet flow rate $F_{i}$ is $2000 \mathrm{~L} / \mathrm{h}$, concentration of oil droplets $C_{\text {oil }}$ is $0.5 \%$ and split ratio $R_{s}$ growing from $2 \%$ to $25 \%$, we can get the separation performance growing from $2.90 \%$ to $98.26 \%$. When concentration of oil droplets $C_{\text {oil }}$ is $1 \%$, we can also obtain a similar rule. From what has been discussed above, when the 
inlet flow rate ${ }^{F_{i}}$ stay constant, the separation efficiency of cyclone will increase as the split ratio increases.

When the split ratio $R_{s}$ is less than $5 \%$, separation efficiency is low and increases slowly as split ratio $R_{s}$ increases. This shows that if the oil droplets size Soil is little enough, oil droplets may flow out though underflow orifice. When the split ratio $R_{s}$ is more than $10 \%$, separation efficiency is still increasing with the increase of split ratio, but the increase degree rise only sluggishly. This indicates that, only a small increase of separation efficiency in influenced by rising split ratio $R_{s}$.

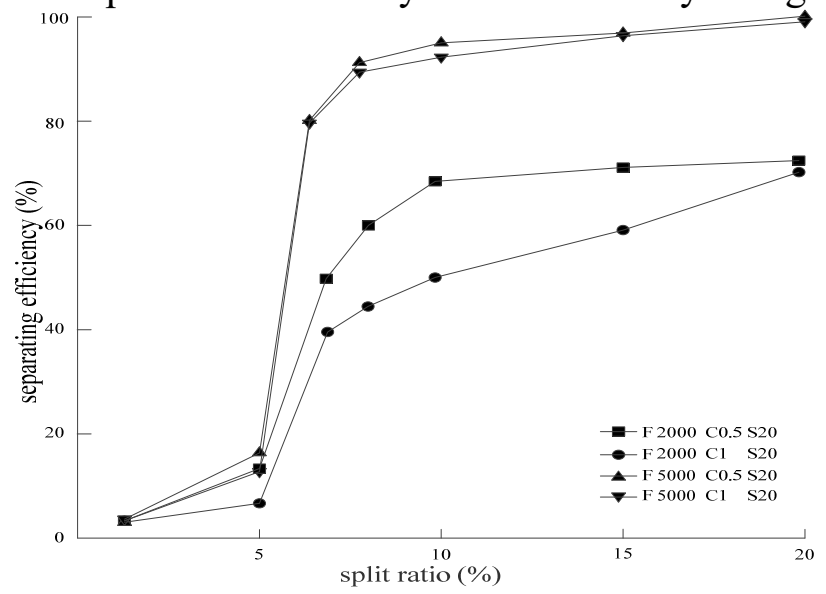

Figure 1. Effect of split ratio on separation efficiency

\section{B. The effect of inlet velocity on separation efficiency}

As shown in Figure2, when the split ratio $R_{s}=10 \%$, inlet velocity ${ }_{i}$ has effect on separation efficiency and pressure. As for oil droplets size $S_{\text {oil }}$ is $20^{\mu m}$ and concentration of oil droplets $C_{\text {oil }}$ is $0.5 \%$, When the inlet velocity $V_{i}$ is growing from $4.37 \mathrm{~m} / \mathrm{s}$ to $20.93 \mathrm{~m} / \mathrm{s}$, the separation performance growing from $69.32 \%$ to $99.75 \%$. As for oil droplets size $S_{\text {oil }}$ is $10^{\mu \mathrm{m}}$ and concentration of oil droplets $C_{\text {oil }}$ is $0.5 \%$, the separation performance growing from $33.65 \%$ to $94.21 \%$. When the concentration of oil droplets $C_{\text {oil }}$ is $1 \%$, there exit a similar rule. When the concentration of oil droplets $C_{\text {oil }}$ is $0.5 \%$ and inlet velocity ${ }_{i}$ is $4.37 \mathrm{~m} / \mathrm{s}$, the threshold of separation corresponds to $15^{\mu m}$ oil droplets size ${ }^{S_{\text {oil }}}$, while inlet velocity ${ }^{V_{i}}$ is $10.46 \mathrm{~m} / \mathrm{s}$, the threshold of separation corresponds to $9.2^{\mu \mathrm{m}}$ oil droplets size ${ }^{S_{\text {oil }}}$.

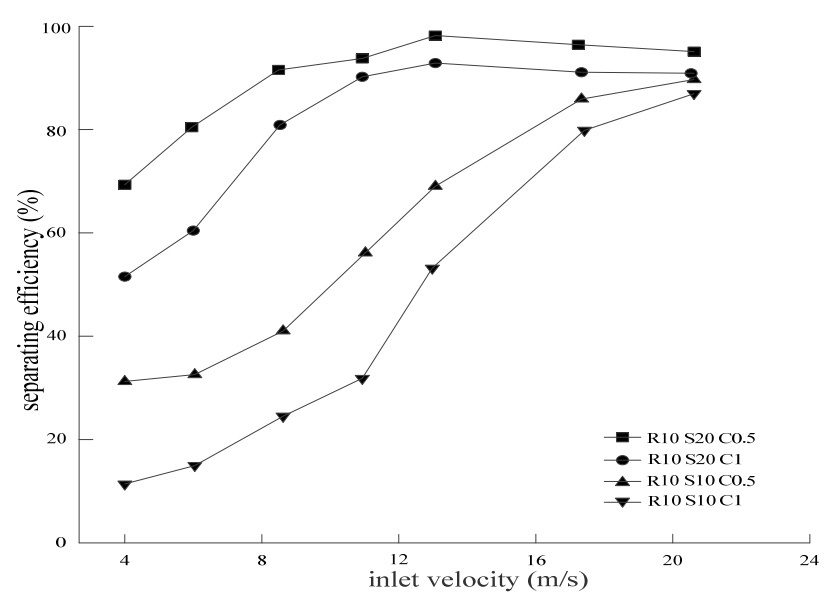

Figure 2. Effect of inlet velocity on separation efficiency 
Inlet velocity ${ }^{V_{i}}$ will lead to tangential velocity increase, thereby increasing driving force of oilwater separation. Although the separation efficiency increases with the increase of flow velocity, inlet velocity increase will enlarge water pressure. All this lead to drop pressure from import to underflow orifice rise, so that operating cost increasing rapidly.

\section{The effect of oil droplets size on separation efficiency}

Generally speaking, different size of oil droplets has different separation efficiency in cyclone separator. Figure 3 simulates separation efficiency of different size of oil droplets under the diversion ratio $10 \%$. For the conditions of inlet flow rate $F_{i}=2000 \mathrm{~L} / \mathrm{h}$ and concentration of oil droplets $C_{\text {oil }}=0.5 \%$, When the oil droplets size $S_{\text {oil }}$ is growing from $5^{\mu \mathrm{m}}$ to $30^{\mu \mathrm{m}}$, separation efficiency will grow from $15.64 \%$ to $92.45 \%$; but for the conditions of inlet flow rate $F_{i}=5000 \mathrm{~L} / \mathrm{h}$ and concentration of oil droplets $C_{\text {oil }}=0.5 \%$, separation efficiency will grow from $26.47 \%$ to $99.76 \%$. When concentration of oil droplets $C_{\text {oil }}$ is $1 \%$, we can also obtain a similar rule. From what has been discussed above, when oil droplets size $S_{\text {oil }}$ is more than $25 \mathrm{um}$, the inlet flow rate $F_{i}$ has less effect on the separation efficiency. So we can take action to increase oil droplets size will improve the separation efficiency.

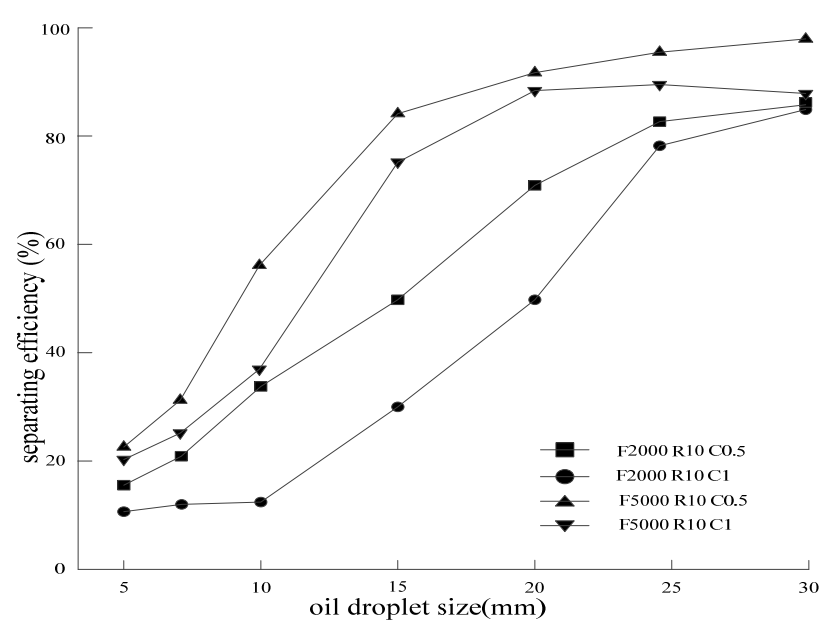

Figure 3. Effect of oil droplets size on separation efficiency

\section{The effect of concentration of oil droplets on separation efficiency}

In the past years, there is little research on the effect of concentration of oil droplets $C_{\text {oil }}$ to separation efficiency. Because the range of oil droplets size $130^{\mu m}<S_{\text {oil }}<150^{\mu m}$ is large, and inlet flow rate $F_{i}$ is quite high, there is less relativity between concentration of oil droplets $C_{o i l}$ and separation efficiency. But for smaller oil droplets size, concentration of oil droplets $C_{\text {oil }}$ will significantly affect the separation efficiency. As shown in Figure 4, when the concentration of oil droplets $C_{\text {oil }}$ is less than $0.5 \%$, separation efficiency almost stay constant as concentration of oil droplets $C_{\text {oil }}$ growing. When the inlet flow rate $F_{i}$ is high enough, such as $5000 \mathrm{~L} / \mathrm{h}$, inlet velocity $V_{i}=11.21 \mathrm{~m} / \mathrm{s}$, and a larger oil droplets size $S_{\text {oil }} 20 \mathrm{um}$, concentration of oil droplets $C_{\text {oil }}$ has a little effect on separation efficiency. But with the inlet flow rate ${ }^{F_{i}}$ or oil droplets size ${ }^{S_{o i l}}$ decreasing, concentration of oil droplets $C_{\text {oil }}$ has obvious influence on separation efficiency. This indicates that the existence of threshold for concentration of oil droplets $C_{\text {oil }}$ is 0.5 , and if less than it, concentration of oil droplets $C_{\text {oil }}$ has little or no effect on separation efficiency. 


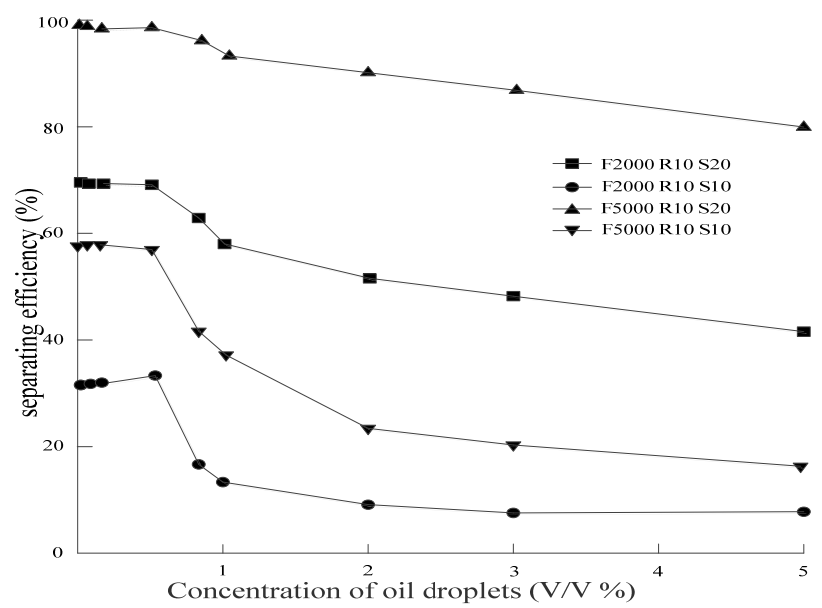

Figure 4. Effect of concentration of oil droplets on separation efficiency

\section{F. The relationship between the simulation results and experimental results}

Concluded from the above discussion, the separation efficiency of the hydrocyclon is affected not only by the operating parameters, such as inlet flow rate and split ratio, but also by material properties, such as oil droplet size and concentration of oil droplets. This effect is very complex.

According to simulation data Figure 1 4, with the statistical software STA-TISTICA6, the relationship is obtained between the separation efficiency and the operating conditions and the material properties by the multinomial fitting method.

$$
\begin{aligned}
& \omega=0.01 F_{i}+12.759 R_{s}-20.703 C_{\text {oil }}+6.133 S_{\text {oil }} \\
& -0.004 R_{s} \times C_{\text {oil }}+0.032 R_{s} \times S_{\text {oil }}+0.325 C_{\text {oil }} \times S_{\text {oil }} \\
& -0.448 R^{2}+1.896 C^{2}-0.077 D^{2}-128.498
\end{aligned}
$$

where: $\omega$ is the separation efficiency for single oil $\operatorname{droplet}(\%), F_{i}$ is inlet flow rate $(\mathrm{L} / \mathrm{h}), R_{s}$ is the split ratio(\%), $C_{\text {oil }}$ is the concentration of oil droplets(\%,V/V), $S_{\text {oil }}$ is oil droplet $\operatorname{size}(\mu m)$. Separation efficiency response to different oil droplet size is calculated according the formula 1 . Then we can calculate the separation efficiency of original water by formula 2.

$$
S E=\sum_{i} \omega_{S_{\text {oil }}(i)} \theta_{i}
$$

Where $S E$ is separation efficiency for all, $\omega_{S_{\text {oil }}(i)}$ is the separation efficiency of $S_{\text {oil }}$ oil droplet size, and $\theta_{i}$ is the volume percent of $S_{\text {oil }}$. The relationship between the simulation results and experimental results is shown as Figure 5. Simulation of separation efficiency is consistent with the experimental results, and the error is within $15 \%$.

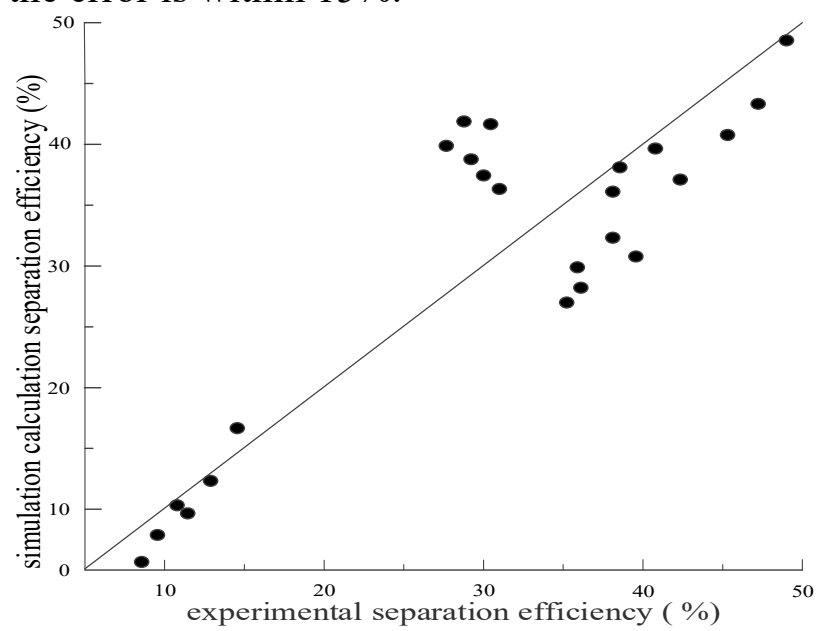

Figure 5. Relationship between the simulation results and experimental results 


\section{Conclusion}

Use computational fluid dynamics which combine Reynolds stress model and Euler multi-phase model together. Discusse the operating conditions and material properties influence the efficiency of oil-water separation of a hydrocyclone by Fluent6.3. The following conclusions are arrived: the hydrocyclone with diameter $50 \mathrm{~mm}$, two inlets, and a single cone angle $5.5^{\circ}$, has an optimum distribution ratio $10 \%$ and a best concentration of oil droplets $0.5 \%(\mathrm{~V} / \mathrm{V})$. Under the above conditions, the hydrocyclone could remove more than $80 \%$ per $15^{\mu \mathrm{m}}$ oil droplets, and the separation of oil droplets cut size is $9.2^{\mu \mathrm{m}}$ for $50 \%$ separation efficiency. Through simulated prediction of separation efficiency corresponding to the measured values, the error is obtained less than $15 \%$

\section{REFERENCES}

[1] Tang B, Xu Y, Song X, et al. Numerical study on the relationship between high sharpness and configurations of the vortex finder of a hydrocyclone by central composite design[J]. Chemical Engineering Journal, 2015, 278:504-516.

[2] Raziyeh S, Ataallah S G. CFD simulation of an industrial hydrocyclone with Eulerian-Eulerian approach: A case study[J]. International Journal of Mining Science \& Technology, 2014, 24(5):643648.

[3] Mokni I, Dhaouadi H, Bournot P, et al. Numerical investigation of the effect of the cylindrical height on separation performances of uniflow hydrocyclone[J]. Chemical Engineering Science, 2015, 122:500-513.

[4] Yang L, Tian J L, Yang Z, et al. Numerical analysis of non-Newtonian rheology effect on hydrocyclone flow field[J]. Petroleum, 2015, 19(1):68-74.

[5] Achari A. High performance MoSmembranes: effects of thermally driven phase transition on COseparation efficiency[J]. Energy \& Environmental Science, 2016, 9.

[6] Achari A, Sahana S, Eswaramoorthy M. High performance MoS2 membranes: effects of thermally driven phase transition on $\mathrm{CO} 2$ separation efficiency[J]. Energy \& Environmental Science, 2016, 9(4):1224-1228.

[7] Mcclure D D, Norris H, Kavanagh J M, et al. Validation of a Computationally Efficient Computational Fluid Dynamics (CFD) Model for Industrial Bubble Column Bioreactors[J]. Industrial \& Engineering Chemistry Research, 2014, 53(37):14526-14543.

[8] Adair D, Bakenov Z, Jaeger M. Building on a traditional chemical engineering curriculum using computational fluid dynamics[J]. Education for Chemical Engineers, 2014, 9(4):e85-e93. 
[9] Mellin P, Kantarelis E, Yang W. Computational fluid dynamics modeling of biomass fast pyrolysis in a fluidized bed reactor, using a comprehensive chemistry scheme[J]. Fuel, 2014, 117(1):704-715.

[10] He J, Zhao Y, Luo Z, et al. Improving separation efficiency of 6-1 mm coal by introducing vibration energy to dense medium gas-solid fluidized bed[J]. Fizykochemiczne Problemy Mineralurgii - Physicochemical Problems of Mineral Processing, 2015, 51(1):95-108.

[11] Bader A J, Afacan A, Sharp D, et al. Effect of liquid-phase properties on separation efficiency in a randomly packed distillation column [J]. Canadian Journal of Chemical Engineering, 2015:1119-1125.

[12] Bhaskar K U, Murthy Y R, Ramakrishnan N, et al. CFD validation for flyash particle classification in hydrocyclones[J]. Minerals Engineering, 2007, 20(3):290-302.

[13] Wang B, Yu A B. Numerical study of particle-fluid flow in hydrocyclones with different body dimensions[J]. Minerals Engineering, 2006, 19(10):1022-1033.

[14] Zhou N, Gao Y, An W, et al. Investigation of velocity field and oil distribution in an oil-water hydrocyclone using a particle dynamics analyzer[J]. Chemical Engineering Journal, 2010, 157(3):73-79.

[15] Regueiro I, Pociask M, Coutinho J, et al. Animal Slurry Acidification Affects Particle Size Distribution and Improves Separation Efficiency.[J]. Journal of Environmental Quality, 2016. 